\title{
Estratégia para o ensino do metabolismo dos carboidratos para o curso de farmácia, utilizando metodologia ativa de ensino
}

\author{
COVIZZI, U.D.S. ${ }^{1}$, LOPES-DE ANDRADE, P.F. ${ }^{2}$
}

${ }^{1}$ Centro Universitário do Norte Paulista, UNORP, São José do Rio Preto, SP.

${ }^{2}$ Universidade Federal Fluminense, UFF, Departamento de Patologia da Faculdade de Medicina, Niterói, RJ. e-mail contato: uderlei@gmail.com

\section{RESUMO}

A metodologia tradicional de ensino tem sido muito questionada sobre o desenvolvimento de competências e habilidades na formação de profissionais na área de saúde. Nesse tipo de metodologia o professor é o principal transmissor do conhecimento, enquanto o aluno assume o papel de espectador passivo. Algumas instituições brasileiras romperam com esse modelo, estruturando a matriz curricular para o ensino centrado no aluno. Alguns cursos de medicina adotaram a Aprendizagem Baseada em Problemas (ABP), uma forma de ensino onde possíveis problemas a serem deparados pelo futuro médico são apresentados para a resolução em pequenos grupos tutoriais. $O$ trabalho propõe utilizar metodologia ativa de ensino-aprendizagem para desenvolver o tema de metabolismo dos carboidratos, conteúdo oferecido na disciplina de bioquímica do curso de farmácia. Dessa maneira, o conteúdo teórico foi apresentado por meio de palestras curtas e objetivas. Posteriormente os alunos foram divididos em grupos tutoriais para a resolução de questões contextualizadas. Durante as atividades, o professor direcionou a discussão para a elucidação das questões. Ao final do módulo os alunos avaliaram a metodologia desenvolvida por meio de um questionário com escala Likert e o conteúdo desenvolvido foi avaliado por uma prova teórica individual. A média aritmética das respostas encontradas indica que os alunos acreditam ter participado ativamente do processo de ensino-aprendizagem, sendo estimulados à discussão e compreensão do tema desenvolvido. As respostas evidenciam uma aproximação maior entre os alunos e o professor tutor. $\mathrm{Na}$ opinião do professor, ocorre maior comprometimento do aluno com a aprendizagem. Conclui-se que uma metodologia inovadora onde o principal responsável pela aprendizagem é o próprio aluno, além de aumentar o interesse em aprender, facilita a aprendizagem pela discussão dos casos em grupo. A contextualização das questões estabelece um estreitamento entre teoria e prática profissional.

Palavras-chave: metodologia ativa, ensino bioquímica, $\mathrm{ABP}$

\section{ABSTRACT:}

The traditional teaching method has been widely questioned on the development of skills and abilities in training healthcare professionals. In the traditional methodology the main transmitter of knowledge is the teacher while students assume passive spectator role. Some Brazilian institutions broke with this model, structuring the curriculum to student-centered learning. Some medical schools have adopted the Problem Based Learning (PBL), a methodology that presents problem questions, to be encountered by future physicians, for resolution in small tutorial groups. Our work proposes to apply an active teaching-learning methodology addressing carbohydrate metabolism during the discipline of biochemistry for under graduation students from pharmacy course. Thus, the academic content was presented through brief and objective talks. Later, learners were split into tutorial groups for the resolution of issues in context. During the activities, the teacher drove the discussion to the issues elucidation. At the end of the module learners evaluated the teaching methodology by means of an applied questionnaire and the developed content was evaluated by an usual individual test. The questionnaire analysis indicates that students believe they have actively participated in the teaching-learning process, being encouraged to discuss and understand the theme. The answers highlight closer ties between students and tutor. According to the professor, there is a greater student engagement with learning. It is concluded that an innovative methodology, where the primary responsibility for learning is centered in the student himself, besides to increase the interest in learning, facilitates learning by cases discussion in groups. The issues contextualization establishes a narrowing between theory and practice.

Keywords: active methodology, biochemistry learning PBL. 


\section{INTRODUÇÃO}

A comparação entre o ensino tradicional de transmissão de conhecimento, onde o professor é o detentor do conhecimento e o aluno é um espectador passivo, com o ensino centrado no aluno, onde o professor participa do processo como um mediador, e o aluno é encarregado de construir o seu conhecimento, tem rendido importantes discussões no cenário nacional da educação [1]. A aula tradicional em si é um típico argumento de autoridade, pois se mantém principalmente através da autoridade do professor, que atua como um fator externo, enquanto que o processo educativo instala-se de dentro para fora.

Quando os dois lados se envolvem em dinâmicas recíprocas, a influência se torna libertadora e não cerceadora [2]. Segundo o modelo tradicional, é considerado como um bom aluno aquele que consegue reter o máximo de informações passadas pelo professor e o bom professor é o bom explicador, que consegue transmitir conteúdos difíceis de modo acessível aos alunos e dessa forma, consegue prender a sua atenção [3].

Segundo Cyrino e Toralles-Pereira [4], a metodologia ativa utiliza-se da problematização como estratégia de ensino-aprendizagem, tendo como objetivo motivar o discente diante do problema, levando-o ao contato com as informações e à produção do conhecimento para solucionar os impasses e promover o seu próprio desenvolvimento. Tratase de uma metodologia libertadora que direciona para uma formação ativa, indicando a necessidade de aprender a aprender [5]. Nesse caso, o ensino é centrado no aluno, a educação é problematizadora, fundamentada no diálogo entre educador e educado, valorizando os conhecimentos colhidos de experiências da vida profissional do docente.

De acordo com Pratt [6], o desenvolvimento da teoria da aprendizagem cognitiva, bem como os resultados de pesquisas desenvolvidas em sala de aula, indica que os estudantes aprendem mais quando podem construir seu próprio conhecimento, como, por exemplo, respondendo questões e resolvendo problemas. Nesse modelo, os conteúdos não são simplesmente apresentados ao aluno de forma acabada, mas sim como problemas a serem descobertos e solucionados [7]. Como facilitadores desse processo de ensino-aprendizagem aparecem os próprios colegas e o professor, que deixa de lado o papel de transmissor de conhecimento e assume o papel de mediador do processo [4].

Essa atitude envolve uma compreensão mais abrangente do processo de aprendizagem, com ênfase dada às ações do aprendiz para aprender o que se propõe. Isso engloba, além dos conteúdos necessários, habilidades, competências, análise e desenvolvimento de valores. Não há como promover essa aprendizagem sem a participação e parceria dos próprios alunos [8].

Schmidt e colaboradores [9] analisaram dez gerações de cursos de medicina de oito universidades holandesas e sugeriram que os alunos graduados por meio da metodologia ativa são mais envolvidos com a aprendizagem e que uma proporção maior de alunos conclui a graduação e na média em um período de tempo menor.

Melhorar a conexão entre parágrafos $O$ uso de novas tecnologias, muito mais interativas usando recursos de informática, é necessário para despertar o interesse em aprender por parte desses aprendizes. Segundo Demo [10], todo ser vivo, de certa maneira "comanda" a sua aprendizagem, já que detém internamente uma dinâmica que atua sempre na posição de sujeito. O aprendiz não só constrói a realidade com que lida, mas principalmente ele se reconstrói. O aluno é natural desse ambiente virtual e isso permite valorizar a sua iniciativa em aprender determinado assunto.

$\mathrm{Na}$ atualidade, observa-se uma forte tendência internacional mostrando a necessidade de mudança na formação profissional para responder aos anseios sociais. Nesse sentido, os programas procuram estimular a educação que valorize a recuperação da dimensão essencial do cuidado, que é a relação entre os seres humanos [4]. $\mathrm{Na}$ vanguarda dessas transformações, encontramos a proposta de alguns cursos de medicina que utilizam a 
Aprendizagem Baseada em Problemas (ABP), mais conhecida pela sua denominação em inglês - Problem Based Learning (PBL).

No caso do ABP, a ênfase pedagógica está centrada na aprendizagem construtiva, constituindo o método mais importante para a instrução de pequenos grupos [11]. Segundo Sakai e Lima (in Berbel [12]), essa metodologia é baseada no estudo de problemas propostos, que direcione para o estudo de um determinado conteúdo. Trata-se de uma metodologia formativa por estimular a participação ativa do aluno em busca do conhecimento.

O professor tutor apresenta o problema, muitas vezes extraído da sua própria experiência profissional, ou da própria literatura, para que os alunos possam propor resoluções para o caso em um determinado período. O ensino-aprendizagem na ABP busca competências, habilidades, atitudes e valores. A aprendizagem centrada no aluno focaliza aprender a aprender e os conhecimentos interdisciplinares [13]. A partir da apresentação, o grupo busca na literatura e na discussão com profissionais informações que auxiliem na elucidação do caso apresentado.

No Brasil, as Diretrizes Curriculares Nacionais (DCN) do curso de medicina romperam totalmente com a metodologia e o currículo tradicional [13]. Cerca de 30 cursos de medicina no Brasil já utilizam o ABP como modelo de ensino-aprendizagem (Universidade Estadual de Londrina, Faculdade de Medicina de Marília, Faculdade Distrital de Medicina de Brasília, Universidade de Ribeirão Preto, Faculdade Christus, Universidade de Fortaleza, Universidade Federal do Ceará, Escola Superior de Ciências da Saúde, Centro Universitário do Espírito Santo, Centro Universitário Vila Velha, Centro Universitário de Anápolis, Pontifícia Universidade Católica de Goiás, Faculdades Integradas Pitágoras, Universidade José do Rosário Vellano, Universidade Anhanguera, Universidade Federal de Mato Grosso, Centro Universitário do Estado do Pará, Universidade do Estado do Pará, Faculdade Pernambucana de Saúde, Pontifícia Universidade Católica do Paraná, Centro Universitário Serra dos Órgãos, Universidade Federal de Roraima, Universidade do Extremo Sul Catarinense, Universidade do Oeste de Santa Catarina, Universidade do Planalto Catarinense, Universidade Anhembi Morumbi, Universidade Tiradentes, Universidade Cidade de São Paulo) [14] e alguns optaram por uma metodologia mista.

O artigo $9^{\circ}$ da DCN [15] de medicina sinaliza um projeto pedagógico de concepção coletiva e centrado no aluno como sujeito da aprendizagem e apoiado no professor como facilitador e mediador do processo ensino-aprendizagem.

O curso de medicina da Universidade Estadual de Londrina, uma das pioneiras na implantação do PBL no Brasil, sugere que a metodologia seja direcionada por um conjunto de sete diferentes passos: $1^{\circ}$ Leitura do problema e identificação de termos, $2^{\circ}$ Identificação dos problemas propostos, $3^{\circ}$ Formulação de hipótese, $4^{\circ}$ Resumo das hipóteses, $5^{\circ}$ Formulação dos objetivos de aprendizagem, $6^{\circ}$ Estudo individual dos assuntos estudados e $7^{\circ}$ Retorno ao grupo tutorial para rediscussão do problema frente aos novos conhecimentos [16].

Os estudos realizados por Gomes e colaboradores [17] analisaram artigos publicados por bibliotecas virtuais no período de 1998 a 2008 e indicaram que a ABP é uma alternativa importante para a implantação da diretriz brasileira para a graduação médica. Outros cursos da saúde já começam a participar dessa transformação. Ceccim e Feuerwerker [18] propõem mudanças na formação dos profissionais de saúde para que o ensino esteja em consonância com as reais necessidades de saúde da população.

Alguns conselhos profissionais, como o de Farmácia (CFF) sinalizam para essa ruptura, propondo uma matriz integradora, onde os cronogramas curriculares estimulariam a forte relação entre os conteúdos teóricos e as atividades práticas, sempre procurando pautar as atividades acadêmicas em valores de caráter ético, sociais, políticos e econômicos [19]. Nessa proposta, os conteúdos de ciência básica, ciências aplicadas, conteúdos profissionalizantes e atividades comunitárias aconteceriam ao longo da formação acadêmica, desde o início do curso [8]. A inserção de atividades profissionais já nos primeiros anos identificaria o aluno com a profissão escolhida desde o início do curso [20]. Essa metodologia busca substituir a transmissão de conteúdos realizada pelo professor tradicional por um 
processo que leve a construção desse conhecimento através da investigação e da integração, criando situações que favoreçam a aprendizagem [21].

\section{JUSTIFICATIVA}

O ensino de bioquímica normalmente ocorre nos primeiros anos dos cursos da área de saúde, fazendo parte da área das ciências básicas [22], sendo que uma parcela desse assunto fora apresentada de maneira superficial ao aluno durante o ensino médio.

Quando questionados sobre algum conhecimento prévio de bioquímica, uma parte significativa dos alunos revelou desconhecer alguns assuntos [23]. A maioria identifica a bioquímica como importante componente curricular para o entendimento de outras disciplinas, como a fisiopatologia [24]. Estudos recentes realizados por Oliveira e colaboradores (2006) [25], efetuados após a conclusão da disciplina de bioquímica, demonstraram que a maioria dos alunos $(82 \%)$ reconhece a sua importância para a sua formação profissional e que esses conhecimentos terão aplicação clínica $(71 \%)$ e que a bioquímica é uma disciplina integradora entre teoria e prática profissional $(69 \%)$.

O ensino do metabolismo usando a metodologia tradicional gera fragmentação do conhecimento e dificulta a integração das vias metabólicas, por induzir o aluno a memorizar as etapas metabólicas. O metabolismo trata de eventos sequenciais [26] e, mesmo que o professor tenha a habilidade para apresentar esse conteúdo como algo a ser entendido e não simplesmente memorizado, a integração de todos os eventos pelo aluno é uma tarefa árdua.

Para promover o aprendizado centrado no aluno como sujeito e principal interessado na aprendizagem, rompendo com o modelo de ensino tradicional através de aulas expositivas, propõe-se uma interação maior entre o conhecimento das vias do metabolismo de carboidratos.

\section{OBJETIVO}

O objetivo desse trabalho foi aplicar e avaliar o emprego de metodologia ativa de ensino-aprendizagem para desenvolver o conteúdo do metabolismo de carboidratos na disciplina de Bioquímica, com alunos acostumados à metodologia tradicional. A avaliação dos valores e competências relacionados à disciplina de bioquímica foi realizada de maneira formativa e somativa.

\section{MATERIAL E MÉTODOS}

Desenvolveu-se a proposta de ensino utilizando metodologia ativa $[4,5,8]$ para alunos do primeiro ano do curso de Farmácia do Centro Universitário do Norte Paulista (UNORP). Realizou-se a atividade durante o período de 16 de agosto a 22 de setembro de 2010.

\section{Procedimentos:}

Desenvolveu-se a atividade em sete etapas diferentes, como apresentado na sequência abaixo:

ETAPA 1 - Apresentação e discussão referente à via glicolítica

O mecanismo da glicólise apresentou-se aos alunos utilizando recursos oferecidos pelo programa PowerPoint e, posteriormente, exibiu-se a animação dessa via através de um recurso multimídia que acompanha o livro Fundamentos de Bioquímica [27], mostrando assim que esse processo tem um desenvolvimento dinâmico e não estático.

Concluindo essa primeira etapa do entendimento do metabolismo da glicose, utilizamos questões-problema integradoras para serem discutidas entre os alunos organizados em grupos menores (grupos tutoriais de até 8 componentes). Nesse momento o professor assumiu o papel de mediador e incentivador da discussão, estimulando a busca do 
conhecimento pelo próprio aluno. Para que a problematização fosse instigadora, as questões, cuidadosamente contextualizadas, buscaram valorizar a capacidade de raciocínio referente ao funcionamento dessa via, o local celular de sua realização, a ausência ou acúmulo de substratos e a presença de possíveis inibidores, sempre correlacionando com situações que o aluno poderá ter a oportunidade de vivenciar na sua vida profissional ou acadêmica (Anexo 1, questões 1 a 3 ).

ETAPA 2 - Atividade prática em laboratório

Por meio do teste de Benedict, que identifica a presença de açúcares redutores, avaliou-se a presença de monossacarídeos livres em diferentes soluções de carboidratos, hidrolisados ou não. O protocolo utilizado foi o descrito por Figueira e colaboradores (2010) [28] com modificações. Após a observação dos resultados qualitativos, os alunos foram capazes de identificar as diferentes soluções apresentadas e sugerir como esses conhecimentos poderiam ser utilizados na interpretação do diagnóstico clínico.

\section{ETAPA 3 - Metabolismo do glicogênio}

Durante essa etapa apresentou-se aos alunos, de uma maneira simplificada, os grupos de transportadores de glicose (GLUT), correlacionando-os com os tecidos e afinidade pela glicose e os processos de glicogênese e glicogenólise, utilizando recursos de multimídia. Após a curta palestra, aplicou-se a problematização através de casos discutidos e resolvidos em grupo (Anexo 1, questões 4 e 5).

ETAPA 4 - A síntese endógena de glicose pelo fígado (gliconeogênese).

A gliconeogênese apresentou-se de maneira sucinta, utilizando recursos de PowerPoint e logo após a apresentação, os alunos agruparam-se e receberam as questões problema para serem discutidas (Anexo 1, questões 6 e 7). Esse questionamento explorou o estado nutricional do indivíduo e a utilização de alguns produtos que interferem nesse processo metabólico (ex. álcool e glicocorticoide).

ETAPA 5 - Controle do metabolismo dos carboidratos

Para o entendimento desse controle, descreveram-se os principais hormônios e suas participações na regulação do metabolismo dos carboidratos. Submeteram-se, em seguida, os grupos de alunos a problematização que envolvia distúrbios metabólicos e hormonais (Anexo 1, questões 8 a 11).

ETAPA 6 - Avaliação somativa e formativa do aluno

Essa etapa foi destinada a avaliação somativa do aluno através de uma prova individual teórica. O questionamento apresentado estimulou o raciocínio e a capacidade integradora dos assuntos, procurando destacar não só as competências, mas também as habilidades desenvolvidas durante as etapas anteriores (Anexo 2).

A avaliação formativa aconteceu ao longo das etapas anteriores, valorizando a participação do aluno nas atividades práticas, discussões em grupo, iniciativa, liderança e desenvolvimento pessoal.

ETAPA 7 - Avaliação do módulo

Ao final do módulo o aluno avaliou a metodologia de ensino, comparando-a com a metodologia tradicional para verificarmos se os objetivos propostos foram atingidos. Também foi feita a auto-avaliação do aluno e do grupo que ele participou.

\section{Análise dos resultados:}

Coletou-se os resultados após a conclusão das etapas propostas, por meio da aplicação de um questionário de avaliação aos alunos. As questões foram formuladas com o propósito de mensurar se os principais objetivos da metodologia ativa haviam sido 
alcançados. Dessa forma, escolheu-se questões que buscam identificar se o módulo desenvolvido estava centrado no aluno como membro ativo, trabalhando em grupos tutoriais e se a atuação do professor era a de mediador do processo ensino-aprendizagem, se o aluno desenvolveu ativamente competências (conhecimento) e habilidades (saber fazer), se o módulo buscou a interação entre o conhecimento e a atuação profissional e se a avaliação realizada tinha caráter somativo e formativo.

Nos resultados, a partir de um questionário com escala Likert, manifestou-se o grau de concordância em dez afirmações, avaliadas por conceitos de um a cinco ${ }^{29}$. Disponibilizou-se um espaço dentro do questionário impresso para que o aluno manifestasse a sua opinião sobre a participação do professor-tutor no processo de ensino-aprendizagem. $O$ objetivo desse espaço foi o de evitar que a opinião pessoal referente ao professor pudesse interferir nos resultados das atividades desenvolvidas.

\section{RESULTADOS E DISCUSSÃO}

Os resultados da avaliação realizada pelos alunos, através do questionário, sinalizaram para uma boa receptividade relativa a metodologia ativa.

As questões propostas, apresentadas na Tabela 1, permitiram a comparação entre as metodologias ativa e tradicional, segundo a opinião dos alunos. Os resultados médios evidenciam que a forma de apresentação do conteúdo não constituiu uma barreira para a participação do aluno nas discussões em grupo, estimulando-o a assumir o importante papel no centro do processo de ensino-aprendizagem.

Além disso, destacou-se que a problematização permite que os mesmos compreendam melhor os temas abordados. O ponto de destaque nessa avaliação foi de uma proximidade maior entre o professor mediador e o grupo de alunos.

Tabela 1. Conceito médio atribuído por 34 alunos referente a questões sobre a metodologia ativa (valoração de 1 a 5$)$.

\section{Questões sobre a metodologia utilizada no curso}

1) A sua participação efetiva no processo de ensino-aprendizagem foi

2) A capacidade de despertar sua participação na discussão em grupo foi

3) A compreensão e execução dos temas abordados foi

4) A proximidade entre professor (tutor) e aluno (aprendiz) foi

5) A sua interação com os outros membros do grupo foi
Conceito médio

3,73

3,68

4,26

4,65

3,85

A Tabela 2 permite análise da opinião do aluno sobre sua própria evolução (questão 1), aplicabilidade do conteúdo estudado (questão 2) e integração com outros assuntos da disciplina de bioquímica (questão 3). Os três itens foram bem avaliados, mas podemos destacar a percepção do aluno na aplicabilidade desse conteúdo, através da problematização, para o seu futuro acadêmico e profissional.

Tabela 2. Conceito médio atribuído pelos alunos referente a questões sobre o conteúdo ministrado (valoração de 1 a 5$)$.

\section{Questões sobre o conteúdo ministrado}

Conceito médio

1) Comparando seus conhecimentos desse conteúdo antes de cursar (conhecimentos do 4,10 ensino médio ou outro curso) o seu progresso foi

2) Você espera que a utilização dos conhecimentos do metabolismo dos carboidratos em outras disciplinas que ainda serão cursadas e na vida profissional seja

3) A integração com outros assuntos discutidos anteriormente foi

A Tabela 3 mostra a relação entre o desempenho individual e o desempenho do grupo. Como pode ser constatado na Tabela 1 , existe uma pequena dificuldade em relação ao 
trabalho em grupo, mas os dados apresentados na tabela 3 indicam que esse inconveniente é individual e que, de maneira geral, o grupo tem um bom desempenho. Os resultados obtidos na Tabela 3 indicam ainda que a melhor maneira de estimular a participação do aluno é através da atividade prática em laboratório (questão 4).

Tabela 3: Avaliação do desempenho individual ou em grupo na opinião dos alunos. (valoração de 1 a 5).

Questão sobre o seu desempenho e o desempenho do seu grupo

1) O seu desempenho durante as aulas e nas atividades desenvolvidas foi

2) $O$ desempenho dos seus colegas de grupo durante as atividades foi

3) O seu aprendizado durante as atividades foi

4) A sua participação na atividade prática no laboratório foi
Conceito médio

3,65

3,97

3,94

4,35

\section{Observações sobre a participação dos alunos feitas pelo professor-tutor}

O uso da metodologia ativa para desenvolver o metabolismo de carboidratos envolve mais o aluno no processo de ensino-aprendizagem. Alguns alunos que participavam passivamente das aulas tradicionais, com pequena ou praticamente nenhuma intervenção, mostraram-se estimulados a integrarem-se efetivamente à discussão propostas.

O envolvimento na aprendizagem, juntamente com os outros colegas e com o professor tutor, gerou a expressão de idéias individuais, que foram utilizadas posteriormente na construção de uma solução coletiva para o problema. Durante a atividade, o aluno desenvolveu as suas competências ao buscar o conhecimento, numa participação efetiva no grupo de discussão e treinou as suas habilidades ao resolver coletivamente as situações problema apresentadas.

\section{CONCLUSÃO}

A metodologia ativa de ensino demonstrou ser uma importante ferramenta para o aprendizado de metabolismo dos carboidratos para o curso de farmácia. A ruptura com a metodologia tradicional, onde o aluno deparava-se com uma série de vias metabólicas individualizadas possibilitou ao aluno visualizar a aplicabilidade desses processos na sua rotina profissional ou acadêmica. Esse tipo de metodologia permite ao aluno opinar livremente durante a discussão em grupo, treinando sua liderança, competência e habilidade, atendendo assim às diretrizes curriculares.

Observou-se que alunos retraídos durante uma aula tradicional, apresentaram e defenderam suas idéias, auxiliando na resolução dos diversos problemas apresentados, ressaltando que a metodologia ativa pode ser desafiadora, instigando o aluno a solucionar os problemas.

Todos os resultados observados indicam um maior aprendizado por parte do aluno, ou seja, por meio desse procedimento o aluno acredita que os conhecimentos (competências) foram melhores estabelecidos do que pela metodologia tradicional.

Outro ponto a ser destacado é a proximidade entre aluno e professor-tutor. Os resultados demonstram que mais de $82 \%$ dos alunos consideram que essa metodologia aproxima o professor tutor do aluno aprendiz.

As respostas dos alunos e as considerações do professor tutor indicam que a utilização de uma metodologia de ensino inovadora, onde o aluno é integrante ativo desse processo, pode ser uma boa estratégia para melhorar aprendizagem referente ao metabolismo de carboidratos, mostrando que outros conteúdos de bioquímica também podem ser desenvolvidos através de metodologia ativa de ensino-aprendizagem. 


\section{REFERÊNCIAS BIBLIOGRÁFICAS}

[1] Zabala A. (1998). A prática educativa: como ensinar. Porto Alegre: Artmed.

[2] Demo P. (2003). Aula: trambique de professor.]

[3] Tavares J, Alarcão I. (2001). Paradigmas de formação e investigação no ensino superior para o terceiro milênio. In: Alarcão I, editor. Porto Alegre: Artmed.

[4] Cyrino E, Toralles-Pereira ML. (2004). Trabalhando com estratégias de ensinoaprendizado por descoberta na área da saúde: a problematização e a aprendizagem baseada em problemas. Cad Saúde Pública;20(3):780-8.

[5] Mitre SM, Siqueira-Batista R, Girardi-de-Mendonça JM, Morais-Pinto NM, Meirelles CAB, Pinto-Porto C, Moreira T, Hoffmann LMA. (2008). Metodologias ativas de ensinoaprendizagem na formação profissional em saúde: debates atuais. Ciênc saúde coletiva; 13(2):2133-44.

[6] Pratt CW, Cornely K. Bioquímica essencial (2006). Rio de Janeiro: Guanabara-Koogan.

[7] Page B. (2010). 12 Things Teachers Must Know about Learning. The education digest. April.

[8] Masetto MT (2003). Docente de ensino superior atuando num processo de ensino ou de aprendizagem. In: Competência Pedagógica do professor universitário. São Paulo: Summus.

[9] Schimidt HG, Cohen-Schotanus J, Arends LR (2009). Impact of problem-based, active learning on graduation rates for 10 generations of Dutch medical students. Medical Education. ;43:211-8.

[10] Demo P. (2009) Aprendizagens e novas tecnologias. Revista Brasileira de Docência, Ensino e Pesquisa em Educação Física. 1:53-75.

[11] Kwan CY (2002). Problem-based learning and teaching of medical pharmacology. Naunyn-Schmiedeberg's Arch Pharmacol. ;366:10-7.

[12] Berbel NAN (1998). A problematização e a aprendizagem baseada em problemas: diferentes termos ou diferentes caminhos? Interface - Comunic, Saúde, Educ. 2:139-54.

[13] Moraes MAA, Manzini EJ (2006). Concepções sobre a aprendizagem baseada em problemas: um estudo de caso na FAMEMA. Rev Bras Ed Med. 30(3).

[14] Escolas Médicas do Brasil. [citado em 2011]; Disponível em:

http://www.escolasmedicas.com.br/metodo.php.

[15] Diretrizes Curriculares Nacionais (DCNs) para o Curso de Medicina. [citado em 2011]; Disponível em: http://portal.mec.gov.br/cne/arquivos/pdf/CES04.pdf.

[16] Metodologia da aprendizagem baseada em problemas. Universidade Estadual de Londrina; [citado em 2012]; Disponível em:

http://www.uel.br/pessoal/moises/Arquivos/APRENDIZAGEMBASEADAEMPROBLEMAS.pdf.

[17] Gomes R, Brino RF, Aquilante AG, Avó LRSD (2009). Aprendizagem Baseada em Problemas na formação médica e o currículo tradicional de Medicina: uma revisão bibliográfica. Rev Bras Educ Med. 33(3).

[18] Ceccim RB, Feurwerkern LCM (2004). Mudança na graduação das profissões e saúde sob o eixo da integralidade. Cad Saúde Publica. 20(5):1400-10.

[19] Uso de Metodologias Ativas de Ensino-aprendizagem em Educação Farmacêutica. [citado em 2011]; Disponível em: http://dnanet.dna.com.br/crfrs/dados/Geraldo.pfd. 
[20] Paranhos VD, Mendes MMR (2010). Competency-Based Curriculum and Active Methodology: Perceptions of Nursing students. Rev Latino-Am Enfermagem.18(1):109-15.

[21] Pimenta SG, Anastasiou LG (2002). Docência e ensino: ensinar a quem? In: Docência no ensino superior. São Paulo: Cortez.

[22] Pinheiro TDL, Silva JA, Souza PRM, Nascimento MM, Oliveira HD (2009). Ensino de bioquímica para acadêmicos de fisioterapia: visão e avaliação do discente. Revista Brasileira de Ensino de Bioquímica e Biologia Molecular. 1.

[23] Zeni ALB (2010). Conhecimento prévio para a disciplina de bioquímica em cursos da área da saúde da universidade regional de Blumenau-SC. Revista Brasileira de Ensino de Bioquímica e Biologia Molecular. 1.

[24] Garrido RG, Araújo TO, Oliveira TH, Guarrido FSRG (2010). O lugar da bioquímica no processo de cuidar: visão de graduandos em enfermagem. Revista Brasileira de Ensino de Bioquímica e Biologia Molecular. 1.

[25] Oliveira RC, lano FG, Silva TL, al e. Percepção dos Alunos do Curso de Odontologia de uma Universidade Brasileira em Relação à Importância da Disciplina de Bioquímica na sua Profissão Revista Brasileira de Ensino de Bioquímica e Biologia Molecular [Internet]. 2006 [citado em ago 2011].

Disponível em: http://www.ib.unicamp.br/lte/bdc/visualizarMaterial.php?idMaterial=346>.

[26] Vargas LHM. A Bioquímica e a Aprendizagem Baseada em Problemas Revista Brasileira de Ensino de Bioquímica e Biologia Molecular [Internet]. 2001 [citado em 19 abr 2012]. Disponível em: http://www.ib.unicamp.br/lte/bdc/visualizarMaterial.php?idMaterial=93>.

[27] Voet D, Voet JG, Pratt CW (2000). Fundamentos de bioquímica. Porto Alegre: Artmed.

[28] Figueira ACM, Sudati JH, Azzolin AS, Rocha JBT (2009). Bioquímica experimental: proposta para ensino de açúcares redutores. PPG em Ensino em Ciências: Química da Vida e Saúde. 1.

[29] Costa PCG (2002). Escala de Autoconceito no Trabalho: Construção e Validação. In: Psicologia: Teoria e Pesquisa. Psic. Brasília, 18(1):75-81. 


\section{ANEXO 1}

\section{QUESTÕES PROBLEMA}

1) Uma senhora deu entrada na emergência de um hospital com a pressão sanguínea abaixo do normal, consequência de uma hemorragia (hipovolemia). A diminuição do volume de sangue diminui a capacidade de distribuição sanguínea (perfusão) para os tecidos. Outro problema detectado foi uma doença pulmonar obstrutiva crônica devido ao hábito de fumar por mais de 30 anos. Os plantonistas notaram que a sua ventilação pulmonar era rápida, a pele estava fria e úmida, os lábios levemente azulados (cianóticos) e a paciente apresentava-se um pouco confusa. Qual poderia ser a relação entre esse caso e a glicólise? Justifique a resposta.

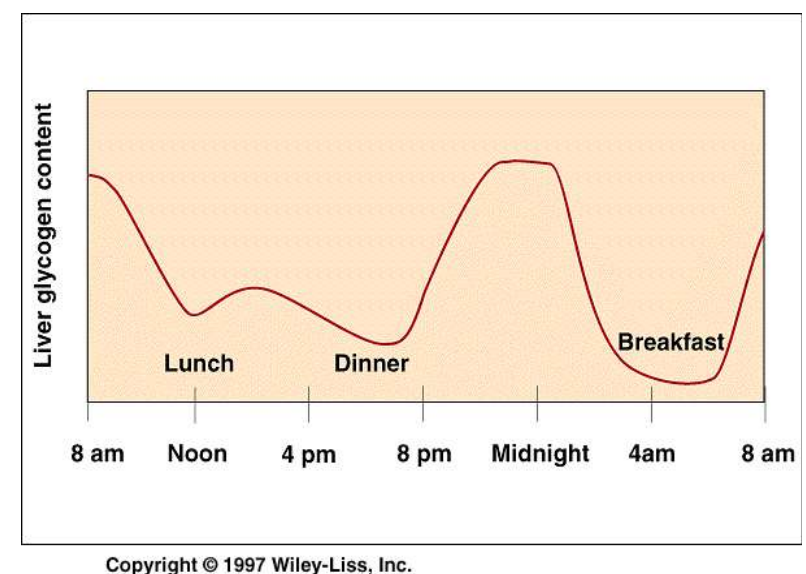

2) Um senhor diabético não se lembrava se havia tomado a dose de insulina das 18:00 h, quando de fato tinha tomado. Acabou decidindo tomar a segunda dose. Quando ele não acordou as $06: 00 \mathrm{~h}$ com o despertador, seus familiares tentaram acordá-lo sem sucesso. Sua filha chamou a ambulância e o senhor foi levado ao hospital em estado de coma devido a baixa concentração de glicose no sangue (37 mg/dL). Qual o motivo da hipoglicemia?

3) O arsenato é uma droga (veneno) que inibe a $6^{a}$ reação da via glicolítica (enzima gliceraldeído 3-fosfato desidrogenase). O que deve acontecer com a via glicolítica em termos energéticos? Justifique a sua resposta.

4) O quadro abaixo mostra a quantidade de glicogênio hepático de uma pessoa durante dia normal de atividade. De acordo com este gráfico responda as seguintes questões:

a) Explique o motivo da alta concentração de glicogênio depois do jantar e baixa concentração depois do almoço.

b) Por que os níveis de glicogênio são tão baixos antes do café da manhã?

c) Como deve estar a atividade da glicogênio fosforilase e da glicogênio sintase as 21:00 h. Justifique a resposta.

5) Uma série de erros inatos do metabolismo, doenças do armazenamento do glicogênio, resultam de deficiências na enzima glicogênio fosforilase. Sabendo-se que a enzima glicogênio fosforilase hepática difere geneticamente da muscular, explique por que um defeito em uma delas pode gerar uma pequena inconveniência enquanto que na outra enzima pode ser fatal.

6) Uma paciente em tratamento com altas doses de um antiinflamatório (dexametasona) para controlar uma infecção pulmonar, voltou ao médico após 5 dias queixando-se de poliúria marcada (aumento na frequência das micções), polidipsia (sede aumentada) e fraqueza muscular. A glicose sanguínea era de $275 \mathrm{mg} / \mathrm{dL}$ (15 mM) sendo o valor de referência 70-100 $\mathrm{mg} / \mathrm{dL}(4,4-5,6 \mathrm{mM})$. Sabendo-se que a dexametasona é um glicocorticóide sintético e que o 
mesmo estimula a proteólise muscular, aumentando o nível de aminoácidos livres e estimulando a gliconeogênese, explique:

a) O motivo da fraqueza muscular.

b) O motivo da hiperglicemia.

7) Um conhecido alcoólatra de uma cidade foi trazido à emergência por um companheiro de boteco. Esse amigo relatou que ele tinha bebido muito na última semana devido a uma discussão com a família e que há praticamente três dias não se alimentava. Estava confuso, trêmulo, agressivo e suando profundamente. Quando a sua pressão estava sendo aferida, ele sofreu uma convulsão. A glicemia medida um pouco antes da convulsão era de $28 \mathrm{mg} / \mathrm{dL}(1,6$ $\mathrm{mM}$ ). Qual a influência do uso abusivo de álcool na taxa glicêmica desse indivíduo?

8) A mensagem que a insulina leva aos tecidos é que a glicose é abundante e pode ser utilizada como fonte imediata de energia ou pode ser convertida em formas de reserva. Como a insulina estimula a utilização de glicose pelos tecidos, onde deve ser imediatamente oxidada ou armazenada para futura oxidação, ela diminui os níveis sanguíneos de glicose, ou seja, a insulina é um hormônio hipoglicemiante. Baseando-se nesse breve relato, responda os itens abaixo:

a) Por que a insulina é produzida pelas células $\beta$ do pâncreas em situações onde a taxa glicêmica é alta?

b) Por que a insulina define que a glicose deve ser utilizada como fonte de energia imediata pelos músculos?

c) Por que a insulina determina que a glicose deve ser armazenada como glicogênio pelo fígado e pelo músculo?

d) Como deve ser a taxa glicêmica de um diabético do tipo I? Por que isso acontece?

9) Em contrapartida a mensagem da insulina, o glucagon leva aos tecidos a informação de que a glicose está baixa e que as fontes de reservas devem ser mobilizadas. Baseado-se nesse breve relato, responda os itens abaixo:

a) Quando as células $\alpha$ do pâncreas vão passar a liberar glucagon?

b) Como o glucagon "avisa" a célula que as reservas devem ser mobilizadas?

c) Qual o papel do glucagon na regulação da glicólise e da gliconeogênese?

d) Como deve ser o nível sérico de glucagon nos diabéticos do tipo I e do tipo II?

e) Responda novamente o item d da questão 8.

10) A adrenalina (epinefrina) é conhecido como o hormônio do estresse, aumentando intensamente o rendimento cardíaco, o fluxo sanguíneo e a mobilização dos substratos energéticos de reserva. Como é a atuação desse hormônio? Podemos afirmar que o seu efeito é o mesmo do glucagon? Justifique sua resposta.

11) O metabolismo dos carboidratos é extremamente regulado, a começar pela entrada de glicose na célula. Sabendo-se que o transporte dessa glicose para o interior da célula depende de um grupo de transportadores (GLUTs) e a utilização dessa glicose depende de uma enzima quinase que adiciona um fosfato a glicose, aprisionando-a na célula, responda sucintamente os itens abaixo, justificando a resposta.

I - Por que o diabético apresenta-se hiperglicêmico

II - Por que o hepatócito só usa glicose quando a sua concentração plasmática for alta? (dois motivos)

III - Por que em normoglicemia o cérebro e as hemácias têm preferência na utilização da glicose? 


\section{ANEXO 2}

\section{AVALIAÇÃO SOMATIVA}

1) O tratamento de uma infecção com o antiinflamatório dexametasona (um glicocorticóide) provocou alterações no paciente, como aumento na freqüência nas micções, aumento na sede e fraqueza muscular. O exame da glicemia revelou que a glicose sanguínea era de quase $300 \mathrm{mg} / \mathrm{dL}$ (normal: $80-100 \mathrm{mg} / \mathrm{dL}$ ). Sabendo-se que o referido fármaco está relacionado ao estímulo do processo de gliconeogênese, podemos afirmar que:

I - A fraqueza observada ocorre devido ao aumento na proteólise muscular que foi estimulada

II - A hiperglicemia é proveniente da produção aumentada de insulina aminoácidos

III - O glicose sanguínea aumentada é proveniente da síntese endógena a partir de adrenalina

IV - O consumo de glicose pelo músculo foi diminuído devido à interferência pela

a) apenas I e II estão corretas

b) apenas I e III estão corretas

c) apenas I, III e IV estão corretas

d) apenas III e IV estão corretas

e) apenas II e III estão corretas

2) A síndrome de McArdle (glicogenose do tipo V) é causada pela deficiência na enzima muscular glicogênio fosforilase. Os portadores dessa doença apresentam dificuldades em realizar exercícios físicos. Seus músculos apresentam um alto teor de glicogênio (entre 2,5 e $4,1 \%$ ), no entanto, seu sangue possui pouco lactato sangüíneo após a liberação de glucagon ou adrenalina, indicando que a glicogênio fosforilase hepática está em seu nível normal. No tratamento para controlar a deficiência deve-se, em primeiro lugar, ter muito cuidado, ou seja, não se deve praticar exercícios físicos vigorosos e deve-se ingerir glicose ou frutose antes de qualquer exercício, mesmo sendo este de pequena intensidade ou pouco vigoroso. Baseado nessas informações, responda:

a) Por que o nível de glicogênio muscular está alto?

b) Qual o motivo da dificuldade na realização de exercícios físicos? Por quê?

c) Explique o tratamento proposto.

3) A quantificação das concentrações sanguíneas de glicose (glicemia) é utilizada para o diagnóstico do diabetes. Estas medições são freqüentemente realizadas nos laboratórios clínicos mais de uma hora após a coleta do sangue. Uma vez que os eritrócitos (hemáceas) são capazes de metabolizar a de glicose, mesmo se o frasco estiver selado (anaerobiose), se faz necessário a utilização de um inibidor da glicólise ( fluoreto que inibe a enzima enolase), para evitar alterações no resultado do exame. Com base nessas informações, assinale verdadeiro $(\mathrm{V})$ ou falso $(F)$.

( ) Se não for utilizado um inibidor teremos uma diminuição na concentração de glicose e um aumento no lactato gliconeogênese

) O fluoreto impede a hiperglicemia devido a inibição concomitante da

) O fluoreto direciona o metabolismo dos eritrócitos para a aerobiose

) Com o fluoreto inibimos a glicólise anaeróbica, que consumiria muita glicose e produziria pouco ATP

glicogênio

) Praticamente toda a glicose livre no sangue será utilizada para a produção do 
4) Pessoas adultas e fisicamente ativas requerem na dieta a ingestão diária de $160 \mathrm{~g}$ de carboidratos e cerca de $20 \mathrm{mg}$ de nicotinamida (precursor do $\mathrm{NAD}^{+}$), lembre-se que cada glicólise consome uma molécula de glicose e um NAD ${ }^{+}$. Baseado nessas informações, podemos afirmar que a diferença tão grande entre as concentrações de glicose e nicotinamida ocorre devido a: (assinale a alternativa correta).

a) A glicose será totalmente metabolizada enquanto que o $\mathrm{NAD}^{+}$será reciclado

b) O NAD' será transformado em lactato e a glicose será preservada

c) A célula é capaz de produzir $\mathrm{NAD}^{+}$no fígado e não é capaz de sintetizar glicose

d) A glicose apenas transporta os elétrons enquanto que o NAD ${ }^{+}$sofrerá oxidação

e) O NAD+ não poderá ser reduzido e nem a glicose será oxidada

5) A regulação de vários processos metabólicos podem ser alostéricos (imediatos) ou hormonal (um pouco mais lento). Esses hormônios são produzidos por glândulas do sistema endócrino e atuam em outros órgãos, como por exemplo o fígado, músculo, tecido adiposo entre outros. De acordo com a capacidade de relacionar-se com alguns fatores e vias metabólicas, assinale as lacunas à esquerda com ( I ) quando se tratar da Insulina, (G) quando se referir ao Glucagon e (A) para a Adrenalina. Observação: nada impede uma via ser ativada por mais de um hormônio.

) Gliconeogênese

) Transportadores GLUT4

) Lipólise

) Metabolismo do glicogênio - glicogênese e glicogenólise

) Lipogênese

) Produção controlada pela glicemia

) Aumento na produção de $\mathrm{AMP}_{\mathrm{c}}$ celular 